\title{
Research on Calibration Method for Digital Energy Meter
}

\author{
Hongwei Xü, Zhan Meng ${ }^{2}$, Junwei Zhang ${ }^{1}$, Chao Ding ${ }^{1}$, Zhongxiao Cong ${ }^{1}$ \\ ${ }^{1}$ Electric Power Research Institute of Guizhou Power Grid Co., Ltd, Guiyang 550000, China \\ ${ }^{2}$ Huazhong University of Science and Technology, Wuhan 430074, China
}

Keywords: calibration; digital energy meter; digital power source; measurement error

\begin{abstract}
As an important part of digital energy measurement system, the measurement accuracy of digital energy meter needs to be calibrated. There are some problems in the existing calibration methods. In this paper, a new calibration method has been proposed, which can be used to calibrate digital energy meter rapidly and accurately. A calibration platform has been built based on this method. By calibrating digital energy meters from different manufacturers in this new method and existing method respectively, it has been proved that the new calibration method can be used to calibrate digital energy meter.
\end{abstract}

\section{Introduction}

With the promotion of intelligent substation, digital energy metering system has been widely used. Digital energy meter is an important part of digital energy metering system, its accuracy would influence the metering performance of whole metering system. The structure of digital energy meter is completely different from traditional energy meter ${ }^{[1]}$, the digital energy meter receives data from merging unit which is in accordance with IEC61850-9-2 protocol, analyses the instantaneous voltage and current sampled value, accumulates the instantaneous power and calculates the electric energy. The calibration equipment and method of traditional energy meter are no longer applicable to digital energy meter.

The existing digital energy meter calibration methods mainly includes standard digital power source method, standard digital energy meter method and watt-second method ${ }^{[2-3]}$. In this paper, all these existing methods have been analysed, some shortcomings of each method have been pointed out. We propose a new method to calibrate the digital energy meter by standard digital energy meter method in combination with Watt-Second method. According to this method, a calibration platform is set up and digital energy meters from different manufacturers have been calibrated

\section{Existing Calibration Method}

Digital energy meter receives IEC61850-9-2 packets from merging unit through optical Ethernet. To calibrate digital energy meter, digital power source is used to generate
ICE61850-9-2 packets, digital energy meter receives these packets from power source directly through optical Ethernet. The accuracy of digital energy meter can be calibrated by comparing standard electric energy with energy represented by electric power pulses of digital energy meter.

\subsection{Standard Digital Energy Meter Method}

Digital power source sends data packets to standard digital energy meter and calibrated digital energy meter through a network switch at the same time. The standard digital energy meter works out the electric energy and outputs standard energy pulses to error calculation module, the calibrated digital energy meter also outputs energy pulses. The error calculation module obtains energy measurement error of calibrated digital energy meter by comparing standard pulses and calibrated pulses. The principle of the calibration method is shown in Fig. 1.

Standard digital energy meter outputs m energy pulses while the calibrated meter outputs $\mathrm{N}$ energy pulses. Since the pulse constant of standard meter and calibrated meter may be different, and these two meters may be of different mode, the energy pulse unit of primary energy mode is imp/MWh, and the pulse unit of secondary energy mode is imp/kWh, it is necessary to covert the number of calibrated energy pulse $\mathrm{N}$ to $\mathrm{m}_{0}$ which is of unit of standard energy pulse.

$$
m_{0}=\frac{K C_{0} N}{C_{L}}
$$

Where $C_{0}$ is the pulse constant of standard digital energy meter, $C_{L}$ is the pulse constant of calibrated digital energy meter, $K$ is the conversion coefficient. If the calibrated meter is of the same mode as the standard meter, $K=1$, if the calibrated meter is of primary energy mode while the standard meter is of secondary energy mode, $K=10^{3} / K_{L} K_{Y}$ ( $K_{L}$ is the virtual current ratio, $K_{Y}$ is the virtual voltage ratio of standard meter), if the calibrated meter is of secondary energy mode while the standard meter is of primary energy mode, $K=10^{-3} / K_{I} K_{U}$ ( $K_{I}$ is the virtual current ratio, $K_{U}$ is the virtual voltage ratio of calibrated meter). 


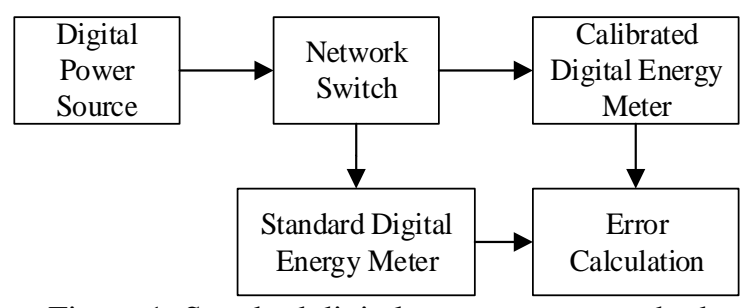

Figure 1: Standard digital energy meter method The energy measurement error of calibrated meter is

$$
\gamma=\frac{m_{0}-m}{m} \times 100 \%
$$

\subsection{Standard Digital Power Source Method}

The digital power source outputs IEC61850-9-2 packets and high frequency electrical energy pulse which is used as the standard electric energy pulse. The calibrated digital energy meter receives the data packets through the network switch and outputs the electric energy pulse to the error calculation module which obtains the energy measurement error of the meter by comparing the standard energy pulse and the calibrated energy pulse. The calibration principle diagram is shown in Fig. 2.

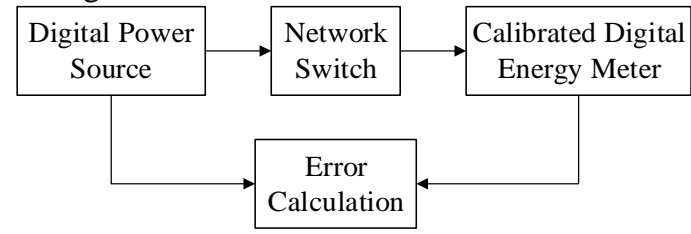

Figure 2: Standard digital power source method In the standard digital power source method, the error calculation module calculates energy measurement error of the meter according to the standard energy pulse and the calibrated energy pulse, and the principle of error calculation is the same as that of the standard energy meter method.

\subsection{Watt-Second Method}

The digital power source outputs IEC61850-9-2 packets to the calibrated digital energy meter which outputs the energy pulse to the pulse acquisition module and the timing module, we can calculate the time $t$ that the calibrated meter outputs $m_{0}$ energy pulses required can be calculated. The electric power generated by the digital power source in time $t$ is converted to $m$ virtual standard electric energy pulses of the meter. The error calculation module obtains the energy measurement error of the meter by comparing the virtual standard electric energy pulse and the calibrated electric energy pulse. The calibration principle diagram is shown in Fig. 3.

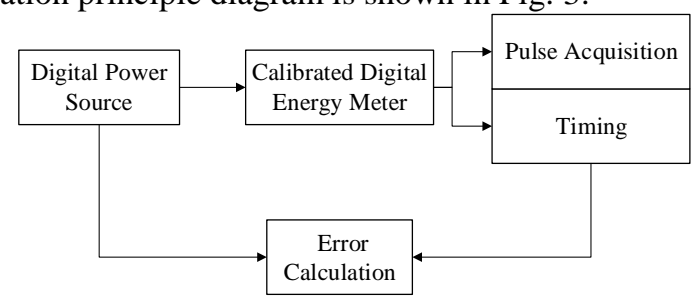

Figure 3: Watt-second method
Set the output power of the digital power source is $P$, the calibrated digital energy meter outputs $m_{0}$ energy pulses in time $t$, The electric energy generated by the digital power source in time $t$ is converted to the virtual standard electric energy pulses under the pulse constant of the calibrated digital energy meter.

There is an equation:

$$
m=K C_{L} P t
$$

Where $C_{L}$ is the pulse constant of the calibrated meter, its unit is imp/MWh, the unit of power $P$ generated by the digital power source is $\mathrm{MW}, K$ is the conversion coefficient, When the calibrated meter is selected the primary power mode, $K=1$, otherwise, the secondary power mode $K=10^{3} / K_{I} K_{U}$, where $K_{I}, K_{U}$ are the virtual current ratio and the virtual voltage ratio of the calibrated digital energy meter respectively.

After completing the conversion, the electric energy measurement error of the calibrated digital energy meter is

$$
\begin{aligned}
\gamma & =\frac{m_{0}-m}{m} \times 100 \% \\
& =\frac{m_{0}-K C_{L} P t}{K C_{L} P t} \times 100 \%=\frac{\frac{m_{0}}{K C_{L} t}-P}{P} \times 100 \%
\end{aligned}
$$

\section{Analysis of Existing Calibration Methods}

When using the standard energy meter method or the standard digital power source method to calibrate the digital energy meter, the error calculation module depends on the standard electric energy pulse and the calibrated electric energy pulse. Since there is no synchronization measure for the start pulse of these two, there is $a \pm 1$ pulse counting error during the time $\mathrm{t}$ at most. The maximum deviation of the calibration is:

$$
\varepsilon=\left|\frac{m_{0}-(m \pm 1)}{m \pm 1}-\frac{m_{0}-m}{m}\right|
$$

If not considering the error of the calibrated meter, $m=m_{0}$, there is:

$$
\varepsilon=\frac{1}{m \pm 1}
$$

When calibrating the 0.2 class energy meter, the error of the required calibration method shall not exceed $0.05 \%$, according to the relationship between measurement error and measurement time, the minimum measurement time corresponding to the pulse constant and the load current of different standard meters can be obtained. When the parameter of calibrated meter is $C_{0}=40000 \mathrm{imp} / \mathrm{kWh}$, $I=5 \mathrm{~A}, U=57.7 \mathrm{~V}$, the calibration of the three-phase fourwire digital energy meter meets the requirement only when the test time be at least $3.46 \mathrm{~min}$. Obviously, these two 
methods are not conducive to the rapid calibration of digital energy meters.

The digital standard source method, compared with the standard energy meter method, its structure is relatively simple and it don't need the standard energy meter. In theory, the packets generated by the standard digital energy source is obtained by hardware calculation, it should be absolutely accurate. However, the packets is in accordance with IEC61850-9-2 protocol, the quantization errors are generated during data framing, In the IEC61850-9-2 packets, the digital quantity representing the sampling value is computed by sampling values and scaling factors.

$$
\begin{aligned}
& u=\operatorname{int}\left(\frac{\sqrt{2} U \sin (\omega t+\varphi)}{q_{u}}\right) \\
& i=\operatorname{int}\left(\frac{\sqrt{2} I \sin (\omega t+\varphi)}{q_{i}}\right)
\end{aligned}
$$

In these formula, $q_{u}, q_{i}$ are the scale factors specified in the protocol. At present, IEC61850-9-2LE protocol is most widely used in real project, which stipulates $q_{u}=0.01$, $q_{i}=0.001^{[4]}$. It is easy to see that the quantization error of the protocol is directly related to the value of the sampled values. According to the simulation results, when the primary voltage is $1 \mathrm{kV}$ and the current is $0.4 \mathrm{~A}$, the quantization error of the digital power source will exceed the error limit of 0.01 class, reaching $0.0134 \%$. According to some relevant standard, in the "current impact test" of the digital energy meter, the error test points should be $1.2 I_{p r}, I_{p r}$ and $0.01 I_{p r}{ }^{[5]}$ ( $I_{p r}$ is the rated primary current of the digital meter).

If the calibrated meter's rated primary voltage is $110 \mathrm{kV}$, rated primary current is $200 \mathrm{~A}$, the minimum current of digital power source would to be $2 \mathrm{~A}$ during the calibration. According to simulation calculation, the energy measurement error caused by the quantization is $0.0013 \%$. Therefore, the electric energy outputted by the digital power source can be used as the standard electric energy in the calibration of digital energy meter. However, there is no standard device for the traceability of digital power source, so this method needs to be studied in the field of traceability.

According to the error calculation formula of watt-second method, time measurement error is an important factor that should not be neglected. Assuming that the time measurement deviation of the pulse acquisition module is $\Delta t$, that is, the actual measured time is $t+\Delta t$, the error of the watt-second method caused by the time measurement error is

$$
\varepsilon=\left[\frac{m_{0}}{K C_{L}(t+\Delta t) P}-1\right]-\left[\frac{m_{0}}{K C_{L} t P}-1\right]
$$

Without considering the error of the calibrated meter, $m=m_{0}=K C_{L} P t$, and $\Delta t<<t$, there is

$$
\varepsilon=\frac{\Delta t}{t}
$$

It can be seen from the above formula, the smaller the error of time measurement is, the smaller the error of watt-second method would be. When the time measurement error is less than $1 \mu \mathrm{s}$ and the test time is $2 \mathrm{~s}$, the error of watt-second method is less than $5 \times 10^{-5} \%$, it can be used to calibrate digital energy meter of $0.2 \mathrm{~S}$ class rapidly.

However, only on the condition that the digital power source is absolutely accurate and steady can the calibration of digital energy meter used watt-second method be accurate. Therefore, the accuracy of watt second method is difficult to be guaranteed in the case of the imperfect traceability chain of digital power source.

\section{Watt-Second \& Standard Digital Energy Meter Method}

In order to solve the problem that the calibration speed of Standard Digital Power Source Method and Standard Digital Energy Meter Method is slow and the calibration accuracy of the Watt-Second Method can't be guaranteed, this paper proposes a new calibration method basing on combination of the watt-second method and the standard digital energy meter method. This new calibration method can obtains the measurement error of the digital energy meter accurately in a short time. The principle of the calibration method is shown in Fig. 4.

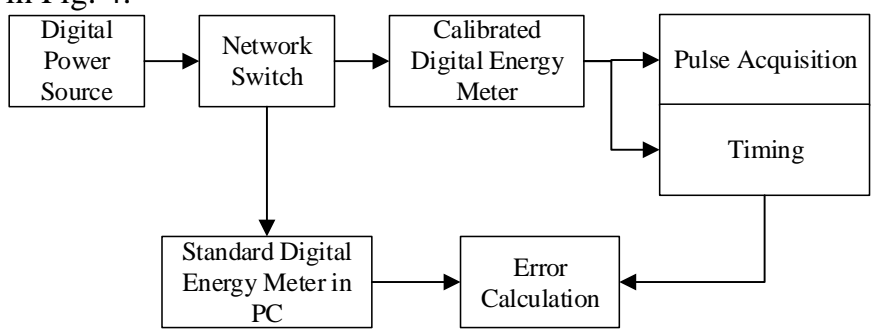

Figure 4: Watt-second digital energy meter method

In this calibration system, the standard channel consist of the digital power source and standard digital energy meter, the calibrated channel consist of the digital power source, calibrated digital energy meter, pulse acquisition module and timing module. Then the PC calculates the error between the standard power and calibrated power.

In this calibration method, the standard digital energy meter is not a physical energy meter, but a standard digital energy algorithm embedded in calibration system in the PC, the input of the standard meter is IEC61850-9-2 packets and the output is standard power $P_{0}$. In a word, the standard digital energy algorithm parses IEC61850-9-2 packets and calculates the real-time power.

The calibrated digital energy meter receives the IEC 61850-92 packets and outputs high frequency pulses of power, the watt-second method is used to calculate the electric energy power. If the calibrated meter outputs $m$ pulses in time $t$, the average energy power of the meter is

$$
P=\frac{K m}{C_{L} t}
$$


In this formula, $C_{L}$ is the pulse constant of calibrated meter; $K$ is the conversion coefficient, when the unit of $C_{L}$ is $\mathrm{imp} / \mathrm{MW} \cdot \mathrm{h}, K=1$, when the unit of $C_{L}$ is $\mathrm{imp} / \mathrm{kW} \cdot \mathrm{h}$, $K=10^{-3} / K_{I} K_{U}, K_{I}$ is virtual current ratio and $K_{U}$ is virtual voltage ratio of the calibrated meter.

Then the error calculation module calculates the energy measurement error of the meter according to the average power of the standard energy meter and the calibrated meter in the time $t$, the calculation formula is

$$
\gamma=\frac{P-P_{0}}{P_{0}} \times 100 \%
$$

Compared with the standard digital power source method and the digital energy meter method, this new method only needs to collect power pulses of calibrated energy meter, does not need to consider the measurement error of unsynchronized pulse. In addition, this method does not use the set power of digital power source as standard power, the standard energy algorithm calculate power directly. The calibration results of this method would not be affected by the accuracy of digital power source, and this method avoids the problem that the digital power source can't be traced. At the same time, this method preserved the speed advantage of watt-second method. This method can certificate digital energy meter quickly and accurately.

\section{Calibration Platform}

According to the calibrate method proposed in this paper to build a digital energy meter calibration platform, calibration device mainly includes digital power source and standard digital energy meter.

\subsection{Digital Power Source}

The standard digital power source is controlled by PC. It can outputs the corresponding IEC 61850-9-2 packets according to the setting parameters.

The digital power source is developed based on FPGA, it can directly calculates the data values in each frame of the sampled value according to the setting parameters. What's more, its error is smaller than the traditional digital power source that based on look-up table method. By verification of the China Institute of Metrology, the relative error of the digital power source's output is less than $0.01 \%$.

\subsection{Standard Digital Energy Meter}

Standard digital energy meter uses the standard digital power algorithm embedded in PC. The computer can run a variety of power algorithms at the same time. The standard digital energy meter contains four standard power algorithms including dot-product-summation algorithm, FFT algorithm, complex Simpson algorithm and complex Cotes algorithm.

At present, most manufacturers produce digital energy meter that uses dot-product-summation algorithm. The dot-productsummation algorithm directly uses the definition of definite integral, and the electric energy is the integration of product of the instantaneous voltage and instantaneous current ${ }^{[6]}$

$$
W=\int_{t_{1}}^{t_{2}} u(t) i(t)
$$

When the process of electric energy's calculation is discretized, the electric energy is expressed as follows:

$$
W=\Delta t \cdot \sum_{n=0}^{N-1} u(n) i(n)
$$

In this formula, $\Delta t$ is the sampling interval, $u(n)$ and $i(n)$ are the voltage and current samples in the sampled value message respectively.

The dot-product-summation algorithm belongs to the rectangular formula in the numerical integration that only has zero order algebraic precision, and it has a large algorithm error for nonstandard sine waves.

The FFT algorithm is often used to calculate the signal frequency, amplitude and phase in the power system. According to the sample value of data packets whose points are fixed, it can quickly calculates the amplitude and phase of the fundamental and the harmonic components, so that it can calculate the average electric power of this sampled packets.

$$
P=\sum_{i=0}^{N} \frac{U_{i} I_{i} \cos \varphi_{i}}{2}
$$

Where $U_{i}, I_{i}$ and $\varphi_{i}$ are the voltage effective value, current effective value and phase difference respectively. $i=0$ represents the DC component, $i=1$ represents the fundamental component.

The total energy is the sum of the electric energy represented by the sampled value packets.

$$
W=\sum_{i=1}^{n} W_{\mathrm{i}}=\sum_{i=1}^{n} P_{i} \Delta t
$$

Where $W_{\mathrm{i}}$ is the energy of each packet segment, and $\Delta t$ is the time represented by each packet segment.

The complex Simpson algorithm and the complex Cotes algorithm are high-order integral numerical algorithm, using interpolation function instead of the integrand to calculate the definite integral approximation. There are $\mathrm{N}$ sampling intervals in the interval $\left[t_{1}, t_{2}\right]$, which means $\mathrm{N}+1$ sampling points, and the complex Simpson formula is used on every two small intervals to obtain the electric energy formula:

$$
W=\left\{\sum_{k=0}^{N / 2-1}\left[\frac{1}{6} p(2 k)+\frac{2}{3} p(2 k+1)+\frac{1}{6} p(2 k+2)\right]\right\} 2 \Delta t_{s}(17)
$$

The computational method of the complex Cotes algorithm is similar to that of the complex Simpson algorithm. In the corresponding sampling interval, the complex Cotes formula is used for every four small intervals, and the formula for calculating the electric energy is: 


$$
\begin{aligned}
W= & \left\{\sum _ { k = 0 } ^ { N / 4 - 1 } \left[\frac{7}{90} p(4 k)+\frac{16}{45} p(4 k+1)+\frac{2}{15} p(4 k+2)\right.\right. \\
& \left.\left.+\frac{16}{45} p(4 k+3)+\frac{7}{90} p(4 k+4)\right]\right\} 2 \Delta t_{s}
\end{aligned}
$$

The complex Simpson algorithm and the complex Cotes algorithm have higher algebraic precision, and the accuracy of the energy calculation can be improved under the same sampling point. The higher the order of the integral algorithm is, the higher the algebraic precision is. But the integral formula is unstable when the order of the integral algorithm reaches 8 . In addition, the higher the order is, the more the system needs to compute, so the general application does not use the high order integral formula.

The calibration system can give the calculation results of the four power algorithms at the same time, which can effectively avoid the influence of the accuracy of the digital power source. At the same time, the energy measurement algorithm of the calibrated meter can be deduced according to the energy measurement error of the calibrated meter under different algorithms.

The relative error of the standard digital energy algorithm embedded in PC is less than $0.01 \%$, which has been verified by the National Institute of Metrology.

\section{Test and Validation}

Five digital watt-hour meters from different manufactures are selected for the test, whose accuracy class for active power is $0.2 \mathrm{~s}$. Parameter configuration of meters is presented in Table I.

\begin{tabular}{|c|c|}
\hline Rated Voltage[V] & $3 \times 57.7 / 100$ \\
\hline Rated Current[A] & $3 \times 1.5(6)$ \\
\hline Rated Frequency[Hz] & 50 \\
\hline Pulse Constant[imp/kWh] & 20000 \\
\hline Sampling Frequency[Hz] & 4000 \\
\hline
\end{tabular}

Table I: Parameter configuration of tested meters According to national standards, the basic error test of digital energy meters is carried out on the test platform constructed in this paper. The voltage of load points tested is $U_{n}$, the current is $0.2 I_{n}, 0.5 I_{n}, I_{n}, 1.2 I_{n}$ and the power factor is $1,0.5$ respectively. Dot-product-summation algorithm is chosen as standard energy metering algorithm. The test results are presented in Table II, showing the error data obtained by using the method proposed in this paper and standard digital power source method to verify digital energy meters.

It can be seen from the test results that the accuracy errors of all the meters tested are far less than the error limit of $0.2 \mathrm{~s}$. The maximum differences between the errors of two method is $0.0025 \%$. Therefore, it can be concluded that "Watt-Second \& Standard Digital Energy Meter method" proposed in this paper can be used to calibrate digital energy meters whose accuracy class is $0.2 \mathrm{~s}$.

\begin{tabular}{|c|c|c|}
\hline \multirow{2}{*}{$\begin{array}{c}\text { Meter } \\
\text { Number }\end{array}$} & \multicolumn{2}{|c|}{ Maximum Error [\%] } \\
\cline { 2 - 3 } & $\begin{array}{c}\text { Watt-Second } \\
\text { \& Standard } \\
\text { Meter Method }\end{array}$ & $\begin{array}{c}\text { Standard Digital } \\
\text { Power Source } \\
\text { Method }\end{array}$ \\
\hline$\# 1$ & 0.0197 & 0.0176 \\
\hline$\# 2$ & 0.0485 & 0.0508 \\
\hline$\# 3$ & 0.0737 & 0.0762 \\
\hline$\# 4$ & 0.0488 & 0.0511 \\
\hline$\# 5$ & 0.0043 & 0.0043 \\
\hline
\end{tabular}

Table II: Test result

\section{Conclusions}

In this paper, the existing calibration methods for digital energy meter is analyzed. In view of the low speed, the imperfect traceability chain and other problems of existing methods, "watt-Second \& Standard Digital Energy Meter method" is proposed, which can calibrate the digital energy meters accurately and quickly. According to the method, the calibration platform is built and several different brands of meters are calibrated, whose results are compared with the calibration results of "standard digital power source method". Finally, it is proved that " watt-Second \& Standard Digital Energy Meter method " can calibrate digital energy meter rapidly. On the other side, the structure of the calibration platform is simple so that it is of high promotion value.

\section{Acknowledgment}

This work is supported by China Southern Power Grid Co., Ltd technology project (No.GZ2014-2-0059), all support is gratefully acknowledged.

\section{References}

[1] Zhou, F., Cheng, Y., Xiao, J., \& Du, J. (2014). Research of digital metering system and calibration technology of smart substation. Advanced Research and Technology in Industry Applications (Vol.241, pp.1240-1242). IEEE.

[2] Tang, Y., Jiang, B., Li, H., \& Chen, X. (2013). Survey on the verification methods for digital electrical energy metering. Transactions of China Electrotechnical Society.

[3] Q/GDW 11018-2013, Particular Requirements for Digital Electricity Meters [S], 2013.

[4] IEC61850 9-2 Specific Communication Service Mapping (SCSM)-Sampled values over ISO/IEC 8802-3[S]. 2004.

[5] T/CEC 116-2016, The Technical Specification for Digital Input Electricity Meter [S], 2016.

[6] Pan, F., Sun, W. M., Zhao, W., Chen, R. M., Xiao, Y., \& Yan, X. U. (2012). Error analysis of numerical integration algorithm in digital power measurement. Electrical Measurement \& Instrumentation. 